\title{
RDUS
}

Revue de DROIT UNIVERSITÉ DE SHERBROOKE

Titre : $\quad$ Y A-T-IL INCESTE? L'EXPERTISE PSYCHOSOCIALE DU TRAVAILLEUR SOCIAL

Auteur(s): $\quad$ René LAPOINTE

Revue : $\quad$ RDUS, 1992-1993, volume 23, numéro 2

Pages: $\quad 375-407$

ISSN : $\quad 0317-9656$

Éditeur : $\quad$ Université de Sherbrooke. Faculté de droit.

URI : $\quad$ http://hdl.handle.net/11143/13400

DOI : $\quad$ https://doi.org/10.17118/11143/13400 
Page vide laissée intentionnellement. 


\section{Y A-T-IL INCESTE? L'EXPERTISE PSYCHOSOCIALE DU TRAVAILLEUR SOCIAL}

par René LAPOINTE*

Nous voulons ici exposer de façon concrète, et à partir d'une expérience à laquelle plusieurs travailleurs sociaux ont participé pendant plus de six (6) ans, la démarche à suivre pour réaliser une expertise psychosociale dans les signalements d'inceste. Nous ferons d'abord voir l'importance de cette expertise, les valeurs qui la sous-tendent, les comportements en cause et les personnes susceptibles d'être impliquées lorsqu'on applique la Loi sur la protection de la jeunesse. Puis, nous ferons la description complète, étape par étape, du processus de l'expertise en l'appliquant à un enfant d'âge scolaire. Nous établirons, par la suite, les distinctions appropriées pour l'enfant non encore scolarisé. Nous terminerons en discutant brièvement de la crédibilité des propos d'agression sexuelle tenus par l'enfant.

Environ 150 situations ont ainsi été expertisées. Dans la nette majorité des cas jugés fondés, l'agresseur a reconnu l'inceste et tous les membres de la famille se sont impliqués dans des thérapies.

Based on the findings of an experiment which lasted more than six years and to which many social workers have contributed, this paper proposes to set out a method to be followed when completing a socio-psychological expertise in cases of incest. It first points out the importance of this expertise, its underlying values, the behaviours involved and the persons likely to be affected by the provisions of the Youth Protection Act. Secondly, it presents a complete step-by-step description of this method as it would be applied in the case of a child of school age. The paper then discusses the appropriate distinctions to be made when pre-school children are concerned. Finally, it discusses what credence is to be given to allegations of sexual assault made by children.

Approximately 150 situations were surveyed by the experts. In a clear majority of cases where it was felt that incest had indeed occurred, the suspected aggressors eventually admitted their actions and all family members later became involved in therapy.

* Travailleur social en pédopsychiatrie au Centre Hospitalier Universitaire de Sherbrooke et chargé de cours à la maîtrise en service social à l'Université de Sherbrooke. 
SOMMAIRE

I. L'IMPORTANCE DE L'EXPERTISE DU DIRECTEUR DE

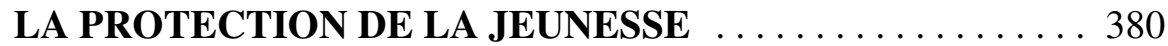

\section{LES VALEURS DE BASE DE L'EXPERTISE}

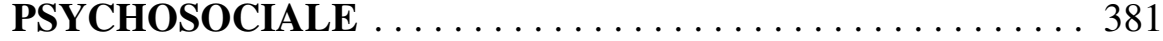

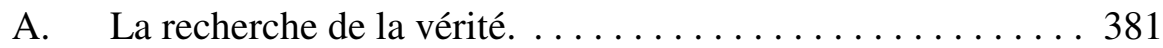

B. L'intégrité physique. .................. 382

C. Le respect des liens familiaux. . . . . . . . . . . . . . 382

D. La croyance au changement de comportement. . . . . . . 382

III. LES CONDITIONS D' APPLICATION DE L'ARTICLE 38(g) DE LA LOI SUR LA PROTECTION DE LA JEUNESSE . . . . 383

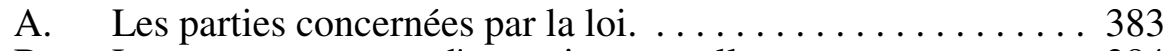

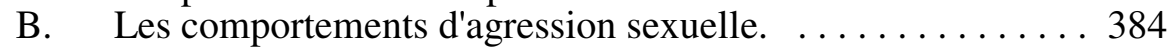

IV. LE PROCESSUS DE L'EXPERTISE PSYCHOSOCIALE . . 384

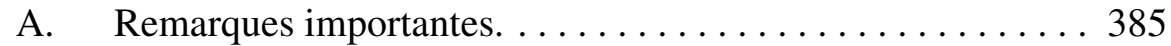

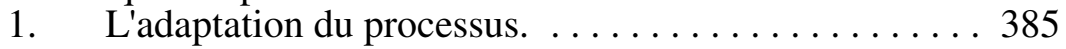

2. L'enchaînement des étapes. . .............. 385

3. La recherche de la vérité et l'aide apportée à la famille.

Les étapes du processus d'évaluation. ............ 386

1. La cueillette des données accessibles. . . . . . . . 387

2. Le contact avec l'informateur. . . . . . . . . . 387

3. La rencontre avec l'enfant agressé et un responsable de

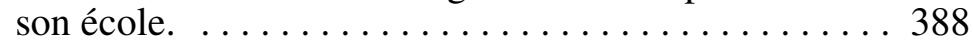

4. La rencontre avec les frères et les soeurs. . . . . . . . 392

5. La rencontre avec la mère. . . . . . . . . . . . . 393

6. L'entrevue avec la mère et l'enfant. . . . . . . . . . . 394

7. La rencontre avec le père. . . . . . . . . . . . . . 395

a) La première phase: «Tisser un lien» . ...... . 395

b) La deuxième phase: «Expliquer les lois» . . . . . 397

c) La troisième phase: la reconnaissance ou la négation de l'agression sexuelle . . . . . . . . . . 399

i) Un long silence. . . . . . . . . . . . . 399

ii) La tentative pour étirer l'entrevue. . . . . . 400

iii) Un non catégorique. . . . . . . . . . . 401

8. La rencontre familiale. ............... 402 


\section{LES PARTICULARITÉS CONCERNANT LES ENFANTS DE}

MOINS DE CINQ ANS ........................ 404

A. La première rencontre avec l'enfant: . . . . . . . . . . . . 404

B. La deuxième rencontre avec l'enfant. .............. 406

C. Les démarches ultérieures $\ldots \ldots \ldots \ldots \ldots \ldots \ldots \ldots 406$

VI. LA CRÉDIBILITÉ DES DÉCLARATIONS DE L'ENFANT .. 407

A. Les facteurs importants dans le contenu du message: . . . . . 408

1. La précision dans la description des gestes d'agression

sexuelle. ....................... 408

2. Les détails périphériques de l'agression sexuelle. . . . 408

3. L'absence de contradiction. ............... 409

4. L'augmentation progressive des agressions sexuelles. . 409

5. Le secret de l'agression sexuelle. . . . . . . . . . . . . 409

6. L'absence de modèle standard lorsqu'il y a plusieurs agresseurs. ...................... 409

B. Les facteurs tirés de l'analyse de la forme du message: . . . . 410

1. Le message principal: l'agression sexuelle. . . . . . . 410

2. L'intensité émotive. . . . . . . . . . . . . . . . . . 410

3. Lorsque l'enfant «protège» l'agresseur. . . . . . . . . . 410

C. Les autres facteurs: . . . . . . . . . . . . . . . . 410

1. Les problèmes de comportement. ............ 411

2. La preuve médicale. .................. 411

3. La deuxième expertise concluante. ..........441

4. Le témoin visuel. ....................441

5. L'aveu de l'agresseur. . . . . . . . . . . . . . 412

\section{LES SITUATIONS OÙ UNE GRANDE PRUDENCE}

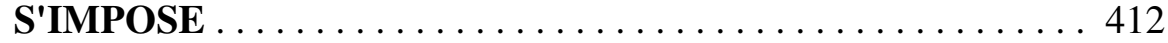

A. Une querelle de couple. . . . . . . . . . . . . 412

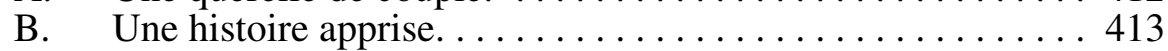

C. Une situation invraisemblable. . . . . . . . . . . . . 413

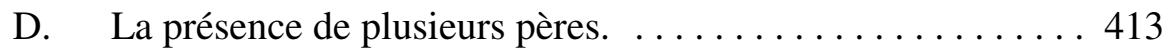

CONCLUSION ............................... 414 


\section{INTRODUCTION}

L'enfant $^{1}$ vient au monde dépourvu de connaissances et de moyens de défense. Contre un milieu souvent hostile, la nature a voulu qu'il puisse s'appuyer sur des parents. Ce sont eux qui lui apportent amour, sécurité et soutien dans sa croissance.

Mais que se passe-t-il lorsque le danger ne vient pas de l'extérieur de la famille, mais de l'intérieur, soit de l'un ou l'autre de ses parents? Et qu'au surplus l'autre parent ne réussit pas à le protéger? Qui blâmer? À qui faire confiance lorsqu'on est trahi et exploité par ceux qui constituent nos racines même dans la vie?

Voilà pourquoi l'inceste n'est jamais une question anodine. Des études ${ }^{2}$ ont montré que la plupart des enfants victimes de telles agressions sexuelles vivent des conséquences importantes, qui peuvent cependant ne se manifester que des années plus tard. L'estime de soi est faussée. La sexualité est éteinte ou exacerbée. Les échecs scolaires, les fugues, les vols, la consommation de drogues, la prostitution, la dépression, l'anorexie ou la boulimie deviennent, à titre d'exemples, des conséquences possibles. Ou encore, le couple se brisera, l'un des conjoints ne pouvant plus faire vraiment confiance à l'autre ni comme partenaire de vie ni comme co-éducateur des enfants.

Heureusement, depuis quelques années, notre société a reconnu ce problème et décidé d'y faire face. Au Québec, on s'est doté de la Loi sur la protection de la jeunesse $e^{3}$, et d'un organisme (régional) chargé de l'appliquer, soit le Centre jeunesse (C.J.).

\section{L'IMPORTANCE DE L'EXPERTISE DU DIRECTEUR DE LA PROTECTION DE LA JEUNESSE}

On réalise de plus en plus que l'évaluation d'un signalement d'inceste repose sur une expertise pouvant avoir de graves conséquences. Toute erreur peut difficilement être réparée.

Prenons un exemple où l'on n'a pas retenu le signalement d'une agression sexuelle pourtant fondée. Il s'agissait d'une fillette de 4 ans agressée sexuellement par son père. L'évaluation, basée essentiellement sur la recherche

1. Le masculin sera utilisé, à moins d'évidence contraire, dans le seul but d'alléger la lecture.

2. Browne, A. et Finkelhor, D. (1986). Impact of Child Sexual Abuse: A Review of the Research. Psychological Bulletin, 99(1), 66-77.

3. L.R.Q., c. P-34.1. 
du profil familial et individuel de l'agresseur, a démontré qu'il ne possédait aucun des traits typiques d'un agresseur et qu'il ne pouvait avoir commis l'inceste. Plus tard, il divorce et recompose une famille avec une femme ayant une fille de 6 ans. Deux ans plus tard, un nouveau signalement est déposé à la protection de la jeunesse. Cette fois, le père finit par avouer qu'il avait effectivement fait des attouchements sur sa fille lors des visites, qu'il avait également eu des gestes déplacés à l'égard de son fils, qu'il commettait le même type d'agression avec la fille de sa deuxième femme. De plus, il a reconnu que la situation décrite lors du premier signalement était bien fondée à cette époque.

Prenons la situation inverse, soit celle où l'on conclut à une agression sexuelle alors que ce n'est pas fondé. Une petite fille de 5 ans, de retour d'une fin de semaine chez son père, semble dire qu'elle a été agressée sexuellement par ce dernier. La mère et la fille rencontrent une intervenante sociale du CLSC, qui s'adresse au directeur de la protection de la jeunesse pour obtenir de l'aide. Sans plus attendre, on avise les policiers qui vont arrêter le père à son travail. Le père nie tout. Peu de temps après, la mère quitte la région avec sa fille et le dossier est transféré au directeur de la protection de la jeunesse de la région de leur nouvelle adresse. On interdit au père de voir sa femme ou ses enfants jusqu'à ce que l'évaluation soit terminée.

Après un délai de 8 mois, l'évaluation est complète. Il s'avère qu'il n'y a pas eu d'agression sexuelle, mais une dramatisation de certains gestes anodins. Même si l'accusation d'agression sexuelle n'était pas fondée, le père a dû changer de village car plus personne ne lui adressait la parole. Et comme tout finit par se savoir, la même situation a persisté dans son nouvel environnement. On pensait qu'il n'y avait pas de fumée sans feu. En outre, ce père et son enfant ont été privés injustement d'une relation normale.

Étiqueter socialement une personne d'incestueuse, c'est la condamner à l'isolement. C'est parfois même faire porter l'opprobre à l'autre parent, qu'on blâme de ne pas avoir protégé son enfant, d'avoir fermé les yeux. Comme s'il devait nécessairement le savoir. Plus que cela, la victime subit parfois le rejet de son entourage.

$\mathrm{Au}$ contraire, ne pas faire cesser une agression sexuelle permet à cette situation de se prolonger et même d'atteindre d'autres enfants. Les conséquences de l'inceste s'en trouvent alors multipliées.

Il ressort que le directeur de la protection de la jeunesse porte une très lourde responsabilité. Voilà pourquoi il doit se donner des moyens adéquats pour déterminer s'il y a eu ou non agression sexuelle. Pour les mêmes raisons, le directeur de la protection de la jeunesse doit veiller à ce qu'on procure aux intervenants, qui le représentent, une formation pertinente pour pratiquer ce type 
d'expertise très complexe, un support clinique adéquat et des conditions propices à un travail de qualité.

\section{LES VALEURS DE BASE DE L'EXPERTISE PSYCHOSOCIALE}

Quatre valeurs de base imprègnent toute l'expertise psychosociale. En effet, on ne peut prétendre vouloir effectuer une expertise de qualité sans rechercher la vérité et la protection de l'intégrité physique de l'enfant, sans avoir le plus grand respect des liens familiaux et sans croire à la possibilité d'un changement de comportement.

\section{A. La recherche de la vérité.}

Le signalement est-il fondé? C'est à cette question fondamentale qu'il faut d'abord répondre puisque tout le reste en découle. Il s'agit toutefois d'une question à laquelle la réponse n'est pas souvent nette, compte tenu que l'agression sexuelle se commet dans l'intimité.

\section{B. L'intégrité physique.}

Il est nécessaire d'être vraiment conscient des graves conséquences que peut avoir l'inceste chez l'enfant agressé sexuellement: perturbation du développement sexuel, diminution de l'estime de soi, difficulté à se respecter et à se faire respecter, dépression, etc. L'agression sexuelle constitue une atteinte à l'intégrité physique et les dommages causés à l'enfant sont souvent plus profonds lorsque l'agression est commise par le père ou la mère.

\section{Le respect des liens familiaux.}

Le travailleur social, comme tout professionnel des sciences humaines, connaît l'importance de la famille pour l'équilibre et le développement de la personne. Aussi, bien que l'enfant soit agressé sexuellement par son père, il a le droit de l'aimer. Bien que la mère doive protéger son enfant, elle a quand même le droit d'aimer son conjoint. Tous ont le droit de désirer vivre ensemble. C'est l'agression sexuelle qu'il faut condamner, non les personnes.

\section{La croyance au changement de comportement.}

Dans les programmes d'aide, il y a très peu de récidives, soit environ $10 \%$. Si on compare cela à bien d'autres problématiques sociales comme l'alcoolisme, les problèmes conjugaux, la délinquance adulte et juvénile, on peut constater que les moyens utilisés sont très adéquats pour aider à modifier les comportements de l'agresseur. D'ailleurs, après le traitement, plusieurs personnes ont fait part qu'elles découvraient enfin le sens de la vie familiale, soit 
un père qui joue son rôle, une mère qui assume le sien et un enfant qui redevient un enfant.

Mais il y a deux conditions essentielles pour que l'aide apportée à la famille réussisse.

Il faut, en premier lieu, que le problème soit reconnu par tous. Dans l'inceste, comme pour toute autre problématique sociale, la reconnaissance de l'existence d'un problème est une condition préalable à tout changement. Nier l'inceste lorsqu'il existe, et à plus forte raison si l'on utilise des moyens légaux pour le faire, c'est pour l'agresseur renoncer à toute possibilité de changement et, pour l'enfant agressé, refuser d'envisager l'inceste avec toutes les conséquences qui peuvent en découler. Enfin, pour l'autre parent, c'est souvent le laisser dans une perplexité déchirante. Cela à tel point que lorsque l'agresseur nie de façon absolue, l'autre parent finit souvent par prendre parti pour l'agresseur, en dépit des expertises ou des jugements établissant le contraire. Dans une telle situation, l'enfant risque grandement un rejet familial, ce qui, on le comprend bien, ne peut qu'intensifier ses difficultés.

L'autre condition, toute aussi essentielle, exige l'acceptation par tous de l'aide proposée. Car il faut bien réaliser qu'une personne ne peut vraiment changer que lorsqu'elle décide volontairement de le faire. Voilà pourquoi, dans le processus d'expertise psychosociale, on attache autant d'importance à la «relation d'aide». Car s'il y a agression sexuelle, il faut d'abord convaincre les gens d'accepter la main que nous allons leur tendre pour les aider à mettre fin à cette situation.

À ces conditions, l'agression sexuelle peut être définitivement enrayée et les conséquences néfastes qui en découlent peuvent être ramenées au moins à un niveau où les gens cessent de souffrir de dysfonctionnements.

\section{LES CONDITIONS D'APPLICATION DE L'ARTICLE 38(g) DE LA LOI SUR LA PROTECTION DE LA JEUNESSE}

La Loi de la protection de la jeunesse $e^{4}$ précise, à l'article 38(g), que la sécurité et ou le développement d'un enfant peuvent être considérés comme compromis lorsqu'une agression sexuelle constitue le motif de compromission. Mais quand peut-on considérer qu'il y a agression sexuelle? Pour répondre à cette question, on doit observer les personnes impliquées et leurs comportements. 


\section{A. Les parties concernées par la loi.}

Les situations d'agression sexuelle visées concernent un enfant et un parent, le parent étant compris dans son sens large: il peut s'agir du père ou de la mère biologique, du beau-père ou de la belle-mère, du conjoint ou de son concubin habituel, des frères et soeurs d'au moins 5 ans plus âgés et tout autre adulte demeurant avec l'enfant.

\section{B. Les comportements d'agression sexuelle.}

Les comportements remis en cause revêtent-ils le caractère d'une agression sexuelle? Si oui, on considérera qu'il y a compromission de la santé et de la sécurité de l'enfant.

Certaines situations ne posent pas de difficultés lorsqu'il s'agit de conclure à un comportement sexuel répréhensif. On pense ici aux pénétrations vaginales ou anales, aux masturbations, aux caresses buco-génitales, et.

Mais, on rencontre souvent des situations moins évidentes lorsqu'il s'agit, à titre d'exemples, d'attouchements, de manipulation des organes génitaux, de caresses sur les cuisses ou les fesses, d'exhibitionnisme, de voyeurisme, etc. Dans ces situations, on doit analyser davantage les faits rapportés pour déterminer s'il s'agit ou non de comportements ayant la nature d'une agression sexuelle.

On parlera de compromission si l'agresseur recherche une stimulation sexuelle pour lui-même ou pour l'enfant ou encore s'il s'en sert pour stimuler un autre partenaire ${ }^{5}$.

\section{LE PROCESSUS DE L'EXPERTISE PSYCHOSOCIALE}

Pour les fins de compréhension du déroulement d'une évaluation standard, nous allons présumer qu'il s'agit d'un enfant d'environ 10 ans. Par la suite, nous adapterons cette évaluation lorsqu'elle concerne un enfant de moins de 5 ans.

5. Lapointe, R. et Mercure, L. (1990). Y a-t-il inceste? Montréal: Stanké, p.26. 


\section{A. Remarques importantes.}

\section{L'adaptation du processus.}

On doit agir différemment selon que l'enfant a atteint l'âge scolaire (5 ans et plus) ou qu'il est en bas âge (moins de 5 ans). Même si le vocabulaire utilisé pour s'adresser à une fillette de 6 ans et une adolescente de 16 ans varie ainsi que les techniques d'entrevue, on suit essentiellement le même processus.

\section{L'enchaînement des étapes.}

Les diverses étapes, qui conduisent progressivement à la preuve d'une agression sexuelle, ne se font pas habituellement la même journée. Ainsi, la cueillette des premières données (étape 1) et le contact avec la personne ayant signalé une possible agression sexuelle (étape 2), peuvent précéder de plusieurs jours les autres étapes. Mais les rencontres avec l'enfant et toutes les parties impliquées doivent avoir lieu la même journée ou, tout au moins, en deux journées consécutives, pour éviter que «le milieu» ne se referme.

\section{La recherche de la vérité et l'aide apportée à la famille.}

Le mandat psychosocial du travailleur social ne peut se limiter à connaître la vérité, bien que cette préoccupation soit essentielle. Le souci de ne pas nuire et, encore plus, d'aider tous les membres de la famille est tout aussi important dans l'expertise faite par le délégué du directeur de la protection de la jeunesse.

Or, si l'agresseur est prévenu de ce qui se passe et, surtout, s'il consulte un avocat avant de rencontrer le travailleur social chargé de l'expertise, la possibilité de nier la commission d'une agression sexuelle augmente considérablement.

Si la négation n'empêche pas de «rechercher la vérité», elle risque cependant de nuire à toute tentative visant à minimiser les conséquences de l'agression sexuelle. Cette négation peut même les aggraver notablement, en provoquant le rejet affectif, et même physique - par le placement en famille d'accueil - de l'enfant identifié alors comme «menteur».

\section{L'ignorance par la mère de l'inceste.}

On entend souvent l'affirmation que la mère est au courant de l'agression sexuelle commise par le père. Notre expérience, ayant porté sur plus de 150 cas évalués en 6 ans, nous autorise à affirmer que dans la très grande majorité des cas, la mère ne sait pas qu'il y a agression sexuelle dans son foyer. De tous les cas évalués, il n'y en a eu que cinq où la mère était au courant. Et, parmi ces cinq situations, trois avaient été signalées par les mères elles-mêmes. 
Il n'est pas rare qu'une mère pense, après coup, qu'il y avait beaucoup d'indices qu'elle n'a pas vus. Toutefois, au moment où elle vivait cela, elle pouvait donner beaucoup d'autres explications à ces signes équivoques ou pouvait encore y voir toutes sortes d'autres causes que l'agression sexuelle. Ainsi, la mère peut penser que: «C'est sa préférée», «Il n'aime que ses filles», «Il veut bien l'éduquer en voulant l'empêcher de sortir avec un ami». Et si, prenant son courage à deux mains, elle ose parler de son appréhension à son conjoint, ce dernier, «scandalisé», pourra l'accuser, par exemple, d'être jalouse de sa fille. Ce qui l'amène à se culpabiliser et à chasser cette idée «folle» de ses préoccupations.

D'un autre côté, bien des enfants sont convaincus que leur mère savait ce qui se passait car ils sont certains de le lui avoir dit. Mais souvent l'enfant, après l'avoir exprimé, l'a nié peu après en laissant à la mère l'impression d'avoir été manipulée. Plus encore, on se rend compte que le message de l'enfant aurait été indirect. Comment sa mère pouvait-elle comprendre, par exemple, que la phrase: «Je n'aime pas que papa nous garde», voulait dire: «Il m'agresse sexuellement»?

\section{B. Les étapes du processus d'évaluation.}

On peut diviser le processus de l'expertise psychosociale en huit étapes distinctes, ce qui permet de minimiser les possibilités d'erreurs ou de conclusions non fondées.

\section{La cueillette des données accessibles.}

Le travailleur social doit d'abord prendre connaissance des renseignements obtenus, lors du signalement de l'agression sexuelle, par la direction de la protection de la jeunesse. On trouve habituellement des informations sur l'enfant, sa famille, son école et sur les faits qui sont à l'origine du signalement.

Dans le dossier, on peut également retracer les coordonnées de «l'informateur», des notes complémentaires au signalement, des copies des signalements et des évaluations antérieures, des jugements des tribunaux, le cas échéant, des indications sur les intervenants antérieurs, etc. Parfois, on y trouve même des rapports sociaux, des évaluations psychologiques, l'évolution de diverses interventions sociales antérieures, ce qui permet de consulter les travailleurs sociaux qui connaissent déjà la famille.

Comme on rencontre de plus en plus fréquemment beaucoup d'agressivité dans ce type d'enquête, il est prudent de relever minutieusement tout indice de violence physique. Ceci peut être déterminant pour choisir le lieu de la rencontre avec les parents et, plus encore, lorsqu'il faut décider si l'enfant va 
demeurer chez lui le soir de la première rencontre ou s'il sera placé en famille d'accueil.

\section{Le contact avec l'informateur.}

Ce contact avec «l'informateur»se fait par téléphone, à moins que la personne ne désire rencontrer le travailleur social chargé de l'enquête.

On doit d'abord s'intéresser à ce qu'il a «vu et entendu». Ce premier constat peut s'avérer très différent de ce qu'il pense et de son interprétation. Par exemple, une personne disait avoir «vu» son gendre pratiquer le cunnilingus sur sa fillette. En distinguant ce qu'elle avait réellement «vu et entendu» de ce qu'elle avait alors pensé, nous avons constaté qu'elle avait seulement interprété des faits. D'ailleurs, le contexte et les révélations de l'enfant ont démontré par la suite qu'il n'y avait pas eu agression sexuelle.

La nécessité de démêler ce qui a été réellement «vu et entendu» de «l'interprétation» doit guider l'intervenant dans ses interventions. Cela est vrai également pour ce que nous livrent les professionnels. En effet, on constate souvent qu'ils exagèrent l'interprétation de certains faits lorsqu'ils croient qu'il y a eu inceste. Par exemple, à un médecin qui ne comprenait pas pourquoi l'enfant n'avait pas été placé dans une autre famille, selon sa recommandation, nous lui avons demandé de fournir un rapport d'évaluation dans lequel il affirmerait que l'enfant était agressé sexuellement. Dès cet instant, le médecin est devenu beaucoup plus nuancé, hésitant même, et il a fini par conclure qu'il ne pouvait pas dire avec certitude qu'il y avait eu agression sexuelle. Dans cette situation, il y avait effectivement d'autres hypothèses pour expliquer ce qu'il avait «vu et entendu».

À cette étape, il est souvent possible d'ajouter d'autres informations utiles en vue de l'expertise. Ainsi, l'informateur peut généralement nous indiquer quelle école fréquente l'enfant agressé ou ses frères et soeurs, où travaillent les parents et leurs horaires. Enfin, comme nous l'avons mentionné, il est utile de s'informer sur la violence possible des parents.

\section{La rencontre avec l'enfant agressé et un responsable de son école.}

Nous tentons de rencontrer l'enfant d'âge scolaire à l'école. C'est un endroit très sécurisant pour lui, éloigné de son domicile et certainement beaucoup plus familier que ne le serait le Centre jeunesse, par exemple. Nous $\mathrm{y}$ allons à la première heure, pour disposer du temps nécessaire au développement qui suivra. 
C'est souvent le directeur de l'école, parfois un professeur qui reçoit l'intervenant social. Celui-ci s'informe du rendement scolaire et du comportement de l'enfant. Nous cherchons ensuite à déterminer si l'on a constaté un changement chez l'enfant ou encore si son rendement scolaire a diminué de façon significative.

Si l'on cherche à connaître les motifs de cette enquête, nous répondons de façon évasive. Si on insiste, il faut alors indiquer clairement qu'il s'agit d'un problème qui ne concerne pas l'école et qu'il est préférable de ne pas en discuter dans l'intérêt de tous.

Par la suite, l'intervenant rencontre l'enfant seul après avoir été introduit auprès de lui par le directeur ou le professeur, afin de le rassurer.

L'entrevue doit d'abord mettre l'enfant en confiance. Cela n'est pas une mince tâche, surtout s'il a désespéré dans l'attente insoutenable de cette rencontre due à une longue liste de cas signalés. La situation reste difficile également du fait qu'on doive rechercher s'il est victime d'agression sexuelle sans poser de questions suggestives, tout en évitant de parler de «tout et de rien», puisqu'on doit pouvoir l'identifier le plus rapidement possible, s'il tel est le cas.

Le travailleur social lui fait donc part de sa fonction et explique à grands traits les objectifs de la Loi sur la protection de la jeunesse $e^{6}$ si l'enfant n'a jamais entendu parler de celle-ci. Puis il dresse avec lui le profil général de chacun des membres de sa famille.

Puis, nous lui demandons si il sait pourquoi nous le rencontrons. Nous nous efforçons pour que l'enfant introduise lui-même le sujet qui nous amène. S'il ne semble pas du tout savoir pourquoi nous sommes là, nous donnons des indices comme: «Tu aurais un problème.»... S'il ne voit toujours pas de quoi il s'agit, nous ajoutons peu à peu: «Un problème qui se passerait chez toi.»... «Avec quelqu'un de ta famille.», «Qui concerne ton père et toi.», etc. À la limite seulement, on lui présente simplement les indices pouvant laisser croire qu'il pourrait y avoir agression sexuelle.

On l'invite à se confier en lui indiquant qu'un travailleur social est une personne qui aide les enfants ayant des problèmes et qu'on a aidé déjà beaucoup d'enfant comme lui. Nous sollicitons également son aide, lui expliquant qu'en dépit de notre volonté de vouloir l'aider, il ne sera pas possible de le faire s'il n'explique ce qui se passe.

6. L.R.Q., c. P-34.1. 
Quand l'enfant se met à parler, nous cherchons à diviser l'entrevue en deux étapes. La première, est consacrée aux faits en lui demandant de raconter ce que nous aurions «vu ou entendu» si nous avions été présents. Il n'est pas toujours possible de procéder ainsi. Lorsque l'enfant est «en crise», il peut vouloir d'abord exprimer immédiatement ce qu'il ressent, ses craintes, sa rage, son désespoir... Il faut alors respecter son rythme et prendre le temps de le réconforter. Quand il redevient plus calme, nous essayons de démêler les faits réels des sentiments.

On l'incite à donner progressivement des détails. Pour cela, il est souvent utile de lui faire suivre une certaine chronologie des événements. Ainsi on peut rattacher l'agression à une période facilement identifiable par l'enfant comme «avant ou après son anniversaire, Noël, durant l'été, la nuit, le jour». Également, on peut l'amener à décrire la disposition des meubles dans la pièce, les vêtements de l'autre, les siens. Quant aux gestes, il faut savoir ceux subis et ceux qu'il a été amené à poser. Il est également important de connaître par quelle stratégie l'agresseur est parvenu à ses fins, et tout particulièrement s'il a usé de force ou de menaces. Enfin, on s'informe si tout cela a été vécu comme un secret et si oui, pourquoi?

La deuxième étape concerne l'expression de ses émotions. Cette façon de procéder a l'avantage de dédramatiser quelque peu la situation puisqu'il a alors déjà parlé de son problème.

A-t-il peur d'être blâmé? Craint-il l'éclatement de la famille? En a-t-il parlé à d'autres personnes ? Si oui, quand et à qui? Qu'a-t-il dit? Quelle a été la réaction de ces autres personnes? On doit donc se préoccuper sérieusement des émotions et des réactions que l'enfant associe à cette situation. Plus particulièrement, on peut lui demander comment il se sent dans la situation présente et à l'égard de l'agression qu'il subit? Croit-il que cela est bien ou non? Qui lui paraît être responsable de ce qui est arrivé? Quelle que soit sa réponse, il faut l'assurer clairement que c'est l'autre qui est responsable de cette situation. Car l'expérience nous a appris qu'il y a souvent là une confusion, source de beaucoup de tourments.

S'il éprouve un sentiment de culpabilité, on doit lui permettre de l'exprimer pour qu'il puisse s'en libérer et mieux comprendre ses attitudes. Puis, on tente de rétablir une pensée plus vraie, lui expliquant que ses réactions - qu'il s'agisse d'acceptation de cadeaux, de soumission sans se débattre, de culpabilité, de recherche de l'attention et de l'affection du père, ou même de plaisir sexuel sont normales mais nullement signe d'un choix libre et éclairé.

Si l'expertise nous mène à penser que le signalement d'inceste est fondé, il faut indiquer à l'enfant qu'on le croit. Autrement, il est préférable de ne rien dire plutôt que de mentir. 
On demande toujours s'il a connaissance que d'autres enfants ont pu être agressés sexuellement, comme lui. De même, on veut savoir s'il craint que son agresseur ne devienne violent en apprenant ce qui arrive. Enfin, nous l'informons qu'il peut signaler sa situation à la police, ce que nous ne ferons pas sans sa permission ${ }^{7}$. S'il décide de le faire, on l'avise que nous pouvons l'accompagner. (Habituellement, les victimes ne désirent pas signaler l'agression à la police, mais elles peuvent vouloir le faire si l'agresseur nie ou refuse de chercher de l'aide.)

Il convient, à la fin, de décrire la forme d'aide qui sera proposée, pour lui, sa mère et son père. Quant à l'agresseur, il faut expliquer à l'enfant que l'aide disponible ne sera possible que si ce dernier reconnaît au préalable son problème. On ne peut changer que ce qu'on reconnaît être un problème pour soi-même.

Si le signalement semble fondé, on l'informe qu'il peut, s'il le désire, être placé temporairement dans une autre famille dès le soir même. Toutefois, s'il court réellement des risques, il sera placé indépendamment de sa volonté ${ }^{8}$. En effet, on doit évaluer si l'enfant ne subira pas des sévices ou un nouvel inceste ou encore s'il peut supporter le «stress» de devoir garder le secret de cette rencontre avec lui jusqu'à ce que d'autres dispositions soient prises. On lui résume, avant de clore l'entrevue, comment les choses vont se dérouler en lui promettant qu'il ne retournera pas seul chez lui après l'école.

\section{La rencontre avec les frères et les soeurs.}

Il est préférable de rencontrer individuellement les frères et soeurs de la victime et, le plus souvent, à leur école également.

Après une entrée en matière semblable à ce qui est décrit ci-haut, on explique que la rencontre a pour but de parler d'un problème familial dont nous leur demandons de parler. Si le frère ou la soeur fait état de l'agression sexuelle, nous l'écoutons en lui faisant préciser les faits dont il a lui-même eu connaissance. S'il ne semble pas avoir une idée du motif de la rencontre, il faut procéder progressivement et lui indiquer d'abord qu'il s'agit d'un problème familial concernant l'une de ses soeurs qui vit des difficultés avec l'un de ses parents.

7. Tout dépendant des régions, il peut exister des protocoles d'intervention selon lesquels on informe systématiquement la police. En Estrie,on ne signale ainsi d'office que les cas où il y a eu violence physique ou encore récidive.

8. Art. 46 et 47 al. 2, Loi sur la protection de la jeunesse, L.R.Q., c. P-34.1. 
On doit éviter de préciser qu'il s'agit d'un signalement d'inceste, sauf s'il s'agit d'un enfant du même sexe que l'enfant agressé et qu'il a atteint l'âge où les agressions sexuelles ont débuté chez l'autre. En effet, dans ce cas il faut alors insister, car il est probable que cet autre enfant puisse également être agressé sexuellement. On l'incite à parler, en lui révélant, qu'à titre d'intervenant, on rencontre beaucoup d'enfants pour les aider et qu'il est très important, pour sa soeur, que nous sachions toute la vérité. Quoi qu'il en soit, nous tentons de vérifier quelques détails périphériques déjà donnés par l'enfant victime, identifié comme victime d'agression sexuelle, comme la description du chalet où ils ont passé l'été; la disposition des meubles dans les chambres où auraient eu lieux des agressions; les habitudes entre sa soeur et son père (par exemple s'ils prennent leur bain ensemble, etc.).

En dernier lieu, on vérifie s'il désire nous faire part d'un problème personnel et s'il y a de la violence physique dans la famille. Et on l'informe qu'on va se revoir plus tard dans la journée.

\section{La rencontre avec la mère.}

Il est préférable de rencontrer la mère seule et la même journée si possible que celle où l'enfant agressé a été rencontré; cela est impératif s'il y a lieu de craindre pour la sécurité de cet enfant.

Après une entrée en matière semblable aux autres rencontres, on arrive à l'objectif de l'entrevue, encore là selon les mêmes modalités que pour toutes les autres rencontres, c'est-à-dire en lui faisant préciser ce dont elle a eu personnellement connaissance.

Si la mère n'est pas au courant ${ }^{9}$ de l'agression sexuelle, il faut alors lui révéler clairement l'objet de la visite. On l'invite alors à réagir. Nous cherchons à faire confirmer des faits communiqués par l'enfant agressé ou les autres enfants. De même, on tente d'ébranler ses efforts pour se convaincre que tout cela n'a jamais pu arriver.

Enfin, on précise à la mère que nous avons déjà évalué un grand nombre de situations, que nous avons procédé ici avec le même souci dans la recherche de la vérité et qu'on doit conclure clairement que l'agression sexuelle a bien eu lieu, (si tel est le cas, évidemment). On lui dévoile ce que nous savons et lui demandons ce qu'elle en pense. Croyait-elle cela possible un jour. Qui va-t-elle croire, l'enfant ou son conjoint?

9. Ce qui, comme nous l'avons dit plus haut, est la situation habituelle. En effet, le parent agresseur sexuel, le père généralement, peut aimer ses enfants et avoir une vie sexuelle harmonieuse avec son conjoint sans que cela n'empêche l'inceste. 
La plupart du temps, la mère est traumatisée par cette révélation. Elle est souvent en état de choc. Elle a besoin de se faire confirmer les faits par l'enfant. D'où l'importance de convoquer l'enfant en même temps afin de rendre possible cette rencontre entre eux, après s'être assuré qu'elle va l'écouter et le soutenir et non le traiter de menteur ou le blâmer. On lui fait comprendre l'importance de son attitude: «L'enfant n'a que deux racines dans sa vie, son père et sa mère. S'il ne peut pas se fier à son père, car il l'agresse sexuellement, il doit au moins pourvoir compter sur sa mère.» Parfois, on doit expliquer quelles peuvent être les conséquences de l'agression sexuelle, surtout quand tant le père que la mère refusent de reconnaître le problème.

Ce n'est qu'une fois la mère calmée, et lorsqu'elle démontre une attitude propice à discuter avec son enfant et à le soutenir, qu'il est opportun de les mettre en présence.

\section{L'entrevue avec la mère et l'enfant.}

On assiste à la rencontre entre l'enfant et sa mère. S'il y a plusieurs enfants victimes, il convient d'offrir à la mère l'alternative de les voir individuellement ou ensemble. Généralement, elle préfère les voir séparément.

Dès le début de la rencontre, il faut rassurer l'enfant en l'informant que sa mère est déjà au courant de la situation, mais qu'elle souhaite qu'il lui raconte lui-même ce qu'il a vécu. On doit participer à la rencontre en les aidant à préciser ce qui est arrivé et à verbaliser ce qu'ils ressentent. Il faut s'assurer que la mère fait confiance à l'enfant et le lui fait clairement savoir. Nous clarifions ensemble pourquoi il ne s'est pas confié à sa mère et qui est le responsable de la situation.

Puis, nous faisant état des services offerts par le Centre jeunesse dans le traitement de l'inceste. Nous le faisons en termes positifs et en insistant sur les deux conditions dont dépend la réussite de l'aide apportée, soit la reconnaissance de l'agression par le père et son acceptation d'être aidé. On rappelle à la mère et à l'enfant qu'ils peuvent signaler la situation à la police et qu'on ne le fera pas sans leur permission (sauf s'il y a eu utilisation de la violence dans l'agression sexuelle ou s'il s'agit de récidive). En dernier lieu, nous indiquons qu'il appartient à l'enfant de décider s'il veut demeurer chez lui cette nuit, sauf si l'on constate un risque évident, auquel cas il sera placé.

Enfin, nous les informons qu'il reste à rencontrer le père et nous leur demandons s'ils croient qu'il va reconnaître avoir agressé sexuellement son enfant, et ce, afin de préparer cette rencontre en cherchant à identifier ses craintes et ses résistances. Ainsi, à titre d'exemples, on peut apprendre qu'il va certainement nier, car la mère a déjà affirmé opter irrémédiablement pour le 
divorce en pareil cas ou parce qu'il craint d'aller en prison ou encore qu'il a peur d'être rejeté de ses autres enfants. On doit s'enquérir également sur l'éventualité de violence physique, ce qui pourra influencer le choix du lieu de rencontre avec lui.

Nous pouvons terminer la rencontre avec la mère en lui demandant si elle croit pouvoir amener le père à reconnaître le problème, notamment en lui faisant comprendre qu'elle croit qu'il a agressé sexuellement son enfant et qu'il est préférable pour lui de dire la vérité. Ou encore en lui disant qu'elle est prête à l'accompagner et à l'aider en autant qu'il reconnaisse son problème, etc. Mais, il arrive souvent que la mère, trop bouleversée par les événements, ne veuille pas le rencontrer.

\section{La rencontre avec le père.}

L'entrevue avec le père ne peut avoir lieu que lorsque l'expertise psychosociale est suffisamment avancée pour permettre de conclure qu'il y a eu agression sexuelle ou encore que le signalement n'est pas fondé. Il est parfois nécessaire de faire d'autres vérifications comme, par exemple, auprès d'un ami de l'enfant agressé, d'une tante, ou encore d'obtenir une évaluation médicale ou psychologique. Prenons pour acquis que l'agression est fondée.

Il n'est pas toujours facile de fixer un rendez-vous avec le père. Après l'avoir informé que le but de la rencontre concerne son enfant, que sa conjointe est au courant, il faut cependant éviter de lui dire qu'il s'agit d'agression sexuelle. S'il résiste, il faut insister en disant, par exemple, qu'il se déplacerait s'il s'agissait d'un accident grave.

L'entrevue avec le père va se dérouler en trois étapes.

\section{a) La première phase: «Tisser un lien»}

Durant la première partie de l'entrevue, on explique au père la Loi sur la protection de la jeunesse s'il ne la connaît pas. Puis, nous lui demandons s'il a une idée de l'objet de cette rencontre.

Parfois, il va aborder immédiatement le problème, auquel cas nous lui demandons des précisions et des explications. Si, au contraire, il nie l'agression sexuelle, nous lui demandons alors ce qui, selon lui, a pu amener son enfant à faire de telles révélations.

Le plus souvent, il détourne la conversation pour éviter d'aborder le sujet comme: «Il a dû encore manquer des cours.» ou encore: «Ne me dites pas qu'il a encore volé!», etc. Nous nous intéressons à ces faits et aux raisons qui font que cela le concerne comme père. Lorsqu'il en a terminé, nous lui faisons 
remarquer que ce n'est pas là l'objet de la rencontre. S'il aborde encore un autre thème, on le laisse faire comme précédemment.

Mais s'il s'inquiète des motifs de la rencontre, on aborde le sujet progressivement comme: «C'est un problème qui se passe à la maison», «Cela concerne votre enfant et l'un de ses parents», «C'est vous le parent en question», «C'est un signalement d'inceste». Chaque fois que nous lui fournissons un nouvel élément, on lui demande ce qu'il en pense.

Durant cette conversation, nous cherchons à connaître son point de vue, à comprendre ses motifs. S'il se dit préoccupé de l'avenir de son enfant, on peut lui indiquer que nous comprenons «qu'il désire donc aider son enfant». Aux efforts déjà effectués antérieurement pour régler les problèmes de son enfant, comme l'absentéisme scolaire, on peut de nouveau insister sur le fait qu'il souhaite voir son enfant réussir. L'objectif de cette prise de contact est importante, pas nécessairement pour ce qu'il dit, mais pour établir peu à peu une relation avec lui. Il doit comprendre qu'on l'écoute, qu'on essaie de le comprendre, qu'on le respecte et que cette rencontre vise à l'aider. C'est de la qualité de cette relation que va dépendre le reste de l'entrevue.

À la fin de cette étape, si l'agression sexuelle n'a pu être abordée, on peut le questionner brièvement sur la façon dont il perçoit son rôle à l'égard de ses enfants, de sa relation avec sa conjointe, avec sa famille d'origine et avec celle de son épouse. On peut chercher à vérifier si la violence physique ou l'agression sexuelle ont existé dans sa famille d'origine ou dans celle de sa conjointe.

Enfin, avant de clore cette partie de la rencontre, on peut lui demander s'il a quelque chose à rajouter. Si oui, on continue l'entrevue. Si non, nous lui indiquons que nous avons maintenant quelque chose à lui dire. Comme nous l'avons écouté, nous désirons qu'il nous écoute à son tour. Sur son approbation, on continue. 


\section{b) La deuxième phase: «Expliquer les lois»}

Nous expliquons alors au père agresseur que l'inceste est couvert par trois lois distinctes, soit par le droit criminel, qui peut conduire à la prison, soit par le droit civil qui pourrait permettre à l'enfant victime d'agression sexuelle de réclamer des dommages ou encore par la Loi sur la protection de la jeunesse ${ }^{10}$, qui concerne la protection de l'enfant. C'est en vertu de cette loi que le travailleur social peut faire enquête ${ }^{11}$ et, dans l'espèce, elle porte sur l'évaluation d'une agression sexuelle.

Si le père nie alors que l'agression sexuelle paraît fondée, il faut alors s'en remettre au tribunal qui décidera s'il y a eu ou non inceste. Par contre, si le père reconnaît les faits et accepte de l'aide, il devient alors possible d'envisager la signature d'une «entente volontaire ${ }^{12}$ » après avoir décrit la forme d'aide offerte comme les thérapies individuelles, de groupes, familiales et conjugales.

On doit, par la suite, insister sur l'importance, en cas d'inceste, que le père le reconnaisse. C'est d'abord essentiel pour lui. On voit souvent que l'inceste continue s'il n'est pas traité. De père incestueux qu'il est aujourd'hui, il deviendra un grand-père «taponneux». Heureusement, ceux qui suivent la thérapie règlent presque tous ce problème. Il s'agit même d'une situation facilement corrigible par la thérapie.

S'il semble plus préoccupé de la survie de son couple, nous reconnaissons qu'il peut arriver qu'elle ne supporte pas ce qui s'est produit et qu'elle se sépare de lui. Mais si elle l'aime et voit qu'enfin il reconnaît son problème et accepte d'être aidé, les probabilités que la famille n'éclate pas sont meilleures. Autrement, comment sa famille peut-elle survivre?

Il faut aussi lui faire comprendre les conséquences sur son enfant. Toutes les recherches démontrent que les enfants agressés sexuellement par leur père vivent de graves conséquences. Le problème résulte du fait qu'on ne sait pas quelles formes elles prendront et quand elles surviendront. Pour un adolescent, ce sera la drogue ou la prostitution. Pour un autre, il s'agira de dépression chronique. Pour d'autres, ce sera l'échec de la relation conjugale ou celui de l'établissement d'une famille. L'aide thérapeutique est surtout efficace lorsque

10. L.R.Q., c. P-34.1.

11. Art. 35.1, Loi sur la protection de la jeunesse, L.R.Q. c. P-34.1.

12. «Entente sur des mesures volontaires»: il s'agit d'un document où le directeur de la protection de la jeunesse résume la situation familiale, le problème, les buts de l'intervention sociale et l'engagement des personnes impliquées à participer à certaines mesures correctives. Signent le père, la mère, l'enfant de 14 ans et plus et le délégué du directeur de la protection de la jeunesse. 
le père reconnaît la réalité des faits. S'il nie l'agression sexuelle, la thérapie a malheureusement une portée limitée pour l'enfant.

Durant l'exposé de la situation par le travailleur social, on doit refuser de se laisser interrompre par les négations du père.

On doit tenir compte des révélations faites par les autres membres de sa famille afin de l'inciter à reconnaître les faits. Ainsi, s'il semble sensible à l'avenir de son enfant, c'est sur cet aspect qu'il faut insister. S'il paraît menacé par la crainte du divorce, on lui indique que la reconnaissance du problème et la demande d'aide ont plus de chances de prévenir le divorce que l'inverse. Si c'est la crainte d'aller en prison qui le conduit à nier, nous devons, à ce stade, lui indiquer qu'on ne le signalera pas à la police. Bien sûr, son épouse ou l'enfant peuvent le faire; mais cela découle habituellement d'un refus de collaborer.

Lorsqu'il semble avoir compris tous les éléments de la situation, il est temps pour le père de connaître la position du travailleur social. Après lui avoir dit être spécialisé dans l'évaluation des signalements d'inceste et avoir procédé ici comme dans les autres cas, il lui dira clairement croire qu'il a agressé sexuellement son enfant. Il lui fera connaître son droit d'être représenté par un avocat et de se défendre. Même plus, il l'incitera à le faire s'il soutient que c'est une erreur. On ne doit jamais admettre de tels faits si ce n'est pas vrai.

Par contre, le travailleur social doit le sensibiliser à l'importance - dans son intérêt - de reconnaître les faits et d'accepter l'aide offerte s'il a ce problème. À défaut d'aide, il doit réaliser que les agressions vont se poursuivre. Son conjoint et son enfant risquent d'en vivre des conséquences désastreuses à long terme, même si ils se font aider en thérapie. On lui précise, enfin, que s'il refuse de reconnaître les faits, le dossier sera transféré au tribunal ${ }^{13}$.

\section{c) La troisième phase: la reconnaissance ou la négation de l'agression sexuelle}

La dernière partie de l'entrevue ouvre la porte à trois possibilités: un long silence; une tentative pour «étirer» l'entrevue; ou encore, un refus catégorique d'admettre l'agression sexuelle.

\section{i) Un long silence.}

Le père ne dit rien et semble réfléchir. Après un temps plus ou moins long d'attente, nous le rassurons en lui faisant comprendre qu'il n'est pas facile de faire une telle admission.

13. Art. 52 al. 3, Loi sur la protection de la jeunesse, L.R.Q., c. P-34.1. 
Lorsqu'il se met à parler, il s'exprime souvent d'une manière générale comme «Oui j'ai un problème avec la sexualité.» Ou encore: «Tout ça est vrai.» De telles généralités sont insuffisantes. Il doit fournir des détails pour mesurer s'il est crédible et s'il est vraiment prêt à dévoiler en entier l'agression sexuelle. L'entrevue se déroule comme avec les autres membres de la famille en distinguant les «faits» des émotions et des interprétations.

Il est parfois tenté de minimiser certains gestes. Il faut l'en empêcher, car il risque de passer tout son temps à «sauver son image» au lieu de l'utiliser pour régler son problème. Il peut aussi tourner autour du problème : «Au début je l'ai touché bien involontairement». Ou encore : «Je ne me rappelle plus si j'ai essayé la pénétration». Il faut se montrer ferme et lui indiquer qu'il vaut beaucoup mieux tout dire, maintenant qu'il a commencé à le faire. On l'encourage à se libérer en lui signalant qu'il se donne, à lui et à son enfant, une nouvelle chance de recréer d'autres liens positifs.

Puis vient le moment d'aborder l'élément «responsabilité». S'il se reconnaît seul responsable, il est opportun de faire état de sa lucidité et de sa franchise. S'il y voit une responsabilité partagée, nous devons lui expliquer pourquoi il ne peut y avoir qu'un seul responsable: lui. Il faut éviter à tout prix de lui permettre de fuir sa responsabilité ou de trouver une excuse pour justifier ses actes. En outre, il doit comprendre que l'enfant n'a pas vraiment la liberté de dire non. Il se croit obligé de participer, même si le père n'utilise pas la violence. Il doit aussi réaliser qu'un enfant recherche l'affection de son père et qu'il ne doit pas confondre cela avec le désir d'une relation sexuelle. De même, lorsqu'une adolescente demande à son père si elle est belle, c'est qu'elle veut se rassurer sur sa féminité, non lui faire des avances.

Enfin, on lui indique qu'il va y avoir une rencontre avec tous les siens et qu'il devra expliquer clairement ce qui s'est passé entre lui et l'enfant. Tous seront là parce qu'ils doivent savoir ce qui se passe au sein de leur famille et que l'enfant agressé sexuellement a le droit à ce que la situation pénible vécue par lui soit reconnue par tous. De plus, le dévoilement familial constitue un excellent moyen de prévenir la récidive. Enfin, le père pourra mieux accepter le traitement en se libérant ainsi de son secret.

\section{ii) La tentative pour étirer l'entrevue.}

L'entrevue peut s'étirer par une discussion plutôt animée. Le père veut bien, par exemple, accepter le traitement, sans toutefois reconnaître qu'il a agressé sa fille. Ou encore, il pose toutes sortes de questions sur les renseignements obtenus des autres personnes et se montre très intéressé à discuter sur ceux-ci, sans jamais se prononcer sur le fondement du signalement de l'agression sexuelle. 
On doit alors se montrer très ferme pour éviter de passer à côté du problème. «On ne peut accepter l'opération à coeur ouvert si on n'a pas de maladie du coeur.» «Il reste une seule question, soit de savoir si oui ou non tu as agressé sexuellement ton enfant.» S'il continue d'utiliser la même stratégie, il faut alors combattre les résistances qui semblent le retenir d'avouer, notamment en cherchant à prévoir ses craintes et à le rassurer, à la lumière de ce que les autres membres de la famille ont déjà dit. On lui rappelle la confidentialité inhérente à la Loi sur la protection de la jeunesse ${ }^{14}$ de sorte que personne ne l'apprendra. Bref, le travailleur social doit se montrer perspicace pour identifier ce qui retient le père d'avouer afin de lui faire comprendre que la situation sera peut-être pire s'il refuse d'avouer et de recevoir de l'aide. Les craintes du divorce, de la police, ou de la prison sont des facteurs qui peuvent facilement amener le père à nier.

Il faut aussi insister sur les avantages que l'enfant en retirera pour briser ses résistances. S'il l'a agressé, il doit maintenant réparer le désastre. La première façon de le faire, c'est de le reconnaître. En effet, l'enfant agressé sexuellement par son père risque de ne jamais vraiment en guérir, si ce dernier nie l'agression.

En cas de négation, nous terminons «à petit pas» l'entrevue. Lentement, nous refermons notre dossier, lui rappelant une dernière fois qu'il peut encore, avant de mettre un terme à l'entrevue, reconnaître les faits, accepter l'aide proposée et signer «une entente volontaire». Dès l'entrevue terminée, l'affaire sera déférée devant le tribunal. Il est arrivé plusieurs fois que le père se ravise alors à la dernière minute.

\section{iii) Un non catégorique.}

Il $\mathrm{y}$ a des situations où le père nie catégoriquement. Il ne faut pas, cependant, confondre ce «non catégorique» avec un «non hésitant». Dans ce dernier cas, nous sommes dans la catégorie précédente. Mais dans l'autre, le père ne veut pas aborder le sujet et même souvent, s'il le peut, il quitte la pièce.

Nous l'avisons alors que le dossier sera transmis au tribunal et qu'il a le droit de se faire représenter par un avocat ${ }^{15}$. Nous l'informons que son enfant sera placé, à moins que la mère ne nous ait fourni auparavant une alternative ou que le père suggère qu'il peut quitter la maison en attendant que la situation soit éclaircie.

14. Art. 11.2, Loi de la protection de la jeunesse, L.R.Q., c. P-34.1.

15. Art. 5, Loi sur la protection de la jeunesse, L.R.Q. c. P-34.1. 


\section{La rencontre familiale.}

S'il y a eu aveu du père, on rencontre, dès que possible, tous les membres de la famille: le père, la mère, l'enfant agressé sexuellement et tous les autres enfants. Tous doivent savoir ce qui se passe, d'autant qu'ils ont subi, souvent, les effets du climat familial incestueux. Lorsqu'ils apprennent l'existence d'un inceste, leur image de l'enfant victime s'en trouve généralement modifiée: ce dernier passe de «mouton noir» à celui qui a souffert. De partager ce secret diminue les risques que les enfants ne se sentent exclus lorsqu'ils verront leur père, leur mère et un seul enfant suivre une thérapie. De plus, comme l'inceste ne peut vivre que dans le secret, briser ce secret familial diminue grandement les risques de récidives et augmente les probabilités que l'enfant victime, étant maintenant cru, parle immédiatement s'il y a récidive. Enfin, cette rencontre est parfois l'occasion de dévoiler d'autres éléments, comme ce fut le cas d'un grand frère qui s'était toujours demandé si sa soeur aînée était adoptée, ce qui était effectivement le cas.

C'est difficile pour le père d'avouer l'inceste et ce ne sera certainement pas facile pour les autres enfants de l'entendre. Il est important que chacun pose toutes les questions qui leur sont utiles pour bien comprendre ce que va dire leur père. Le travailleur social peut lui-même poser quelques questions pour clarifier la situation. Il n'est pas nécessaire de donner tous les détails, mais il faut que toute la famille sache de quoi l'on parle, qui est la victime et jusqu'où l'agression a été poussée.

La situation de l'inceste étant suffisamment dévoilée, on aborde la question de la responsabilité. Il doit être clairement établi pour tous, que le père est le seul coupable. Si l'enfant agressé, ému par les circonstances, tente d'excuser son père, il faut réfuter ses arguments et affirmer catégoriquement que seul le père est responsable. On doit inciter la mère à donner ouvertement son opinion à ce sujet. Le père doit être incité à parler en ce sens. Tant que cette question n'est pas comprise et acceptée par tous, il faut continuer d'en parler.

Les membres de la famille doivent s'interroger sur les raisons pour lesquelles le secret fut si longtemps caché. Il en ressort la plupart du temps que l'éloignement entre la mère et l'enfant victime a empêché ce dernier de se confier. D'où la nécessité pour la mère de comprendre qu'elle doit développer des liens plus étroits avec cet enfant. Parfois, ce sont des menaces paternelles, même voilées, qui ont bloqué les confessions de l'enfant. On doit alors signaler clairement que toute nouvelle tentative pour inciter la victime à ne plus parler et à se dédire aboutirait obligatoirement devant le tribunal. Enfin, il faut rappeler que la violence physique est toujours inacceptable et sera intolérable en pareil cas. 
La rencontre familiale terminée, on peut enfin envisager de signer l'entente sur mesures volontaire ${ }^{16}$. La première partie décrit le problème le plus clairement possible. La rédaction est faite en leur présence en disant tout haut ce qu'on veut écrire, obtenant ainsi leur assentiment avant de le faire.

La deuxième partie comprend les objectifs visés par l'intervention sociale, à savoir la cessation de tout acte d'agression sexuelle, la prévention de la récidive, la réparation des dommages et la protection de l'enfant victime.

La dernière partie spécifie les moyens à prendre pour remédier à la situation. Tous s'engagent à suivre les rencontres individuelles, les rencontres de groupe, les thérapies familiales et même conjugales proposées. Parfois, il faut prévoir des clauses particulières comme l'obligation pour la mère de ne pas laisser son enfant seul en présence du père, l'obligation pour le père de se soumettre à une évaluation psychologique ou psychiatrique, ou encore l'obligation pour l'enfant agressé de suivre une thérapie individuelle ou de subir un examen médical. S'il y a un placement de l'enfant, on indique la durée et les modalités.

Chaque personne concernée doit lire l'entente et la signer. Chacun en reçoit une copie, les invitant à contacter le travailleur social, au besoin, avant qu'un autre intervenant du Centre jeunesse ne prenne en charge leur dossier.

\section{LES PARTICULARITÉS CONCERNANT LES ENFANTS DE MOINS DE CINQ ANS}

La différence la plus importante, lorsque l'expertise concerne un enfant âgé de moins de 5 ans, résulte de la chronologie des rencontres. En effet, le travailleur social devrait rencontrer d'abord le parent non soupçonné, et d'autres personnes significatives si nécessaire, avant de rencontrer l'enfant. En ce cas, la mère est le plus souvent au courant du signalement, quand elle ne l'a pas initié elle-même. À l'aide de ces rencontres préalables, on prend des informations sur ce que l'enfant a pu dire, sur ses gestes pour le dire, sur ses connaissances en matière de sexualité et son vocabulaire à ce sujet. On doit aussi s'enquérir si l'enfant est de contact facile et de la manière dont on peut arriver à mieux lier connaissance avec lui?

16. Art. 52, Loi sur la protection de la jeunesse, L.R.Q., c. P-34.1. Voir à ce sujet, BOISCLAIR, C. «L'entente sur les mesures volontaires dans la Loi sur la protection de la jeunesse», (1982) 13 R.D.U.S. 143 à 210. 


\section{A. La première rencontre avec l'enfant:}

Elle a généralement lieu à son domicile, dans sa chambre. Au préalable, nous avons demandé à sa mère quel moment de la journée était le plus propice pour le rencontrer, en évitant les périodes de ses siestes et de ses émissions favorites. Il est important d'apporter un ou deux livres expliquant à l'aide d'images la sexualité aux enfants, des poupées sexuées, un bloc à dessins et des crayons, le tout à l'abri des regards de l'enfant.

Après avoir été introduit par la mère auprès de l'enfant, il est souhaitable de demeurer seul avec lui, sauf si cela l'indispose. En ce cas, on demande à la mère de ne jamais répondre (ni en parole, ni en geste), à la place de l'enfant.

Le travailleur social lui explique qu'il rencontre beaucoup d'enfants comme lui afin de les aider. Si l'enfant s'ouvre rapidement sur son problème, on l'écoute attentivement. Si non, on doit essayer de l'y amener graduellement, sans cependant introduire le sujet.

Pour créer un climat favorable, on peut amener l'enfant à parler, en le questionnant sur son âge, sur la maternelle, sur les adultes de son entourage, sur ses amis, ses jouets préférés, ses émissions favorites, etc. tout en refusant d'accepter des gestes comme réponses de façon à ce qu'il s'exprime verbalement pour se faire comprendre. Quand il s'habitue à parler plutôt qu'à gesticuler, on peut lui demander:

- $\quad$ de dire ce qu'il aime le plus et le moins en chacun des adultes qu'il connaît;

- $\quad$ de dessiner les membres de sa famille et d'expliquer son dessin. Par exemple, une enfant de trois ans, ayant dessiné son père sur une feuille à part et mit beaucoup de monstres autour de lui, expliqua qu'elle ne l'aimait pas parce qu'il lui «chauffait les fesses» avec de la «crème chauffante» et son «gros doigt».

On peut éventuellement utiliser des livres sur la sexualité expliquée aux enfants pour saisir les mots avec lesquels l'enfant désigne les parties de son corps et délimiter les connaissances qu'il a sur le sujet. Parfois, cela l'amène à parler des éléments du signalement, mais il faut éviter de lui demander s'il a déjà vécu ce qu'il voit sur les images.

On peut aborder aussi la question du secret, spécialement si les informations obtenues en ont fait mention, en lui demandant s'il sait ce qu'est un secret, si lui-même a déjà eu un secret. On peut lui indiquer que les secrets ne doivent jamais être révélés, sauf à quelqu'un qui nous veut du bien et veut nous aider. Si l'enfant veut parler en secret du secret, il faut lui permettre de le faire 
à voix basse ou de se cacher sous le lit ou encore d'utiliser son téléphone jouet ou faire parler ses poupées.

Durant cette première entrevue, on ne doit pas dévoiler encore l'objet du signalement. On se contente de favoriser indirectement un échange pour que, si l'agression sexuelle existe, il arrive à en parler de lui-même. S'il y parvient, on l'amène à préciser tous les faits «vus ou entendus» à partir de ce qu'il raconte. Aussi, s'il dit que son papa lui met «un gros doigt» dans ses fesses, on peut l'inviter à reproduire l'acte à l'aide de ses poupées. A l'occasion, on peut utiliser les poupées sexuées.

Parfois, l'enfant cesse de parler soudainement et il ne veut manifestement plus continuer cette conversation. À ce moment, il est préférable de terminer la rencontre en lui indiquant qu'on aimerait bien revenir jouer avec lui.

\section{B. La deuxième rencontre avec l'enfant.}

Si la première rencontre n'a pas porté fruit, il faut en changer l'approche en choisissant un moment et un endroit propice à l'intimité, que ce soit à la maison ou à la garderie et en le rencontrant si possible le même jour ou le lendemain matin.

Cette fois, nous inversons les rôles et disons à l'enfant qu'on a besoin d'aide. Utilisant le même vocabulaire que celui de l'enfant, nous abordons le sujet qui nous préoccupe, sans cependant être précis. On peut dire, par exemple: «J'ai besoin qu'on m'aide à comprendre quelque chose. On m'a dit qu'il se passait quelque chose quand tu vas chez papa les fins de semaine. Veux-tu en parler?»

Ses propres poupées deviennent alors un moyen utile pour mimer les situations en cherchant constamment à lui faire préciser ce qu'il exprime et à distinguer entre les faits réels de ce qu'il pourrait s'imaginer. Si l'enfant évoque des scènes à caractère sexuel, on peut alors utiliser les poupées sexuées pour l'aider à bien préciser tout ce qu'il dit. Ses mots et ses gestes sont extrêmement révélateurs.

\section{Les démarches ultérieures}

Lorsque la seconde entrevue ne permet pas de recueillir des indices d'une agression sexuelle, il y a lieu de fermer le dossier. Mais si quelques doutes persistent, nous poursuivons l'expertise psychosociale dans un autre lieu et en l'absence voulue de la mère, puisque l'enfant est maintenant habitué à ces rencontres. 
Il est souvent nécessaire de requérir un examen psychologique pour le tout jeune enfant présumé agressé. D'une part, cette dernière expertise aide à vérifier certaines hypothèses comme celles où l'enfant parlerait d'agression sexuelle pour «plaire à sa mère». D'autre part, elle contribue à nous éclairer sur la relation qu'entretient l'enfant avec ses parents et comment il vit la situation d'agression sexuelle. Elle viendra aussi corroborer les recommandations ultérieures en cas de déni de l'agresseur.

L'évaluation médicale peut parfois être utile. Elle est même toute indiquée s'il y a crainte d'infection, de maladie vénérienne ou si l'on pense qu'il $\mathrm{y}$ a eu relation sexuelle. Il est prudent de faire appel à un pédiatre ayant une certaine expertise dans ce genre de problème.

\section{LA CRÉDIBILITÉ DES DÉCLARATIONS DE L'ENFANT}

Dans un ouvrage récent, l'auteur Hubert Van Gijseghem ${ }^{17}$ souligne que le taux de fausses allégations, selon les études récentes de Faller ${ }^{18}$, de Jones \& McGraw $^{19}$ et de Mans ${ }^{20}$ se situe entre $1 \%$ et 3\% seulement. Plus réservé, John C. Yuille ${ }^{21}$ parle d'un taux de véracité de «plus de 90\%». Quoi qu'il en soit des statistiques, c'est la situation concrète de l'enfant évalué qu'il faut juger.

À la lumière de notre exposé, on comprend que c'est essentiellement l'analyse de ce que dit l'enfant qui permet de conclure qu'il a été, ou non, agressé sexuellement. D'ailleurs, affirme une psychologue spécialisée dans la problématique de l'inceste:

«aucun test psychologique n'a été spécialement conçu pour prouver l'existence de l'inceste... On n'a pas encore trouvé non plus un type de réponse à un test projectif particulier qui serait un indicateur fiable. ${ }^{22}$

D'abord, il faut s'attarder au contenu, mais aussi à la manière dont l'enfant livre son message. Néanmoins, quelques autres facteurs peuvent nous aider.

17. Gijseghem, H.V. (1992). Particularité du témoignage de l'enfant victime d'abus sexuel. Dans Gijseghem, H.V. (1992). L'enfant mis à nu. L'allégation d'abus sexuel: La recherche de la vérité. Montréal : Méridien psychologie, p. 19.

18. Faller, K. (1988). Child sexual abuse. New York: Columbia University Press.

19. Jones, D. et McGraw, J. (1987). Reliable and fictious accounts of sexual abuse in children. Journal of Interpersonal Violence, 2, 27-45.

20. Mann, E. (1985). The assessment of credibility of sexually abused children in criminal court case. Americam Journal of Forensic Psychiatry, 6, 9-15.

21. Yuille, J.C. (1992). L'entrevue de l'enfant dans un contexte d'investigation et l'évaluation systématique de sa déclaration. Dans Gijseghem, H.V. (1992). L'enfant mis à nu. L'allégation d'abus sexuel: La recherche de la vérité. Montréal: Méridien psychologie, p. 101.

22. Lapointe, R. et Mercure, L. (1990). Y a t-il inceste? Montréal: Stanké, p.121. 


\section{A. Les facteurs importants dans le contenu du message :}

\section{La précision dans la description des gestes d'agression sexuelle.}

L'enfant agressé sexuellement peut, même très jeune, donner des détails surprenants concernant les actes sexuels. S'il a vécu un ou plusieurs événements qui l'ont beaucoup marqué, il peut décrire avec précision la position et les gestes de l'autre, les siens, etc. Cela a d'autant plus de poids que l'enfant démontre des connaissances nettement avancées pour son âge, surtout si ses parents reconnaissent qu'il n'a que peu de connaissances sur la sexualité.

Par exemple, une fillette de cinq ans qui, de l'avis de ses parents, n'aurait pas de notions sur la sexualité, n'aurait pas pu décrire aussi précisément les étapes d'une masturbation, si elle ne l'avait pas vécu, en disant : qu'elle prend le pénis entre son pouce et ses doigts, qu'elle le touche de bas en haut, que le pénis devient dur, qu'il bouge tout seul, qu'il devient très humide et «qu'après il devient tout petit». Il n'y a que l'apprentissage par expérience qui a pu lui enseigner cela.

\section{Les détails périphériques de l'agression sexuelle.}

Il est étonnant de constater combien un enfant, même jeune, peut donner beaucoup de détails périphériques comme le vêtement qu'il portait, celui que portait l'autre, l'endroit où s'est déroulée la scène, ce qu'ils faisaient auparavant, ce qu'ils ont fait après, où était la mère, la couleur de l'auto, etc. Tout est important pour vérifier ses allégations. Par exemple, cela permet de recouper les souvenirs de la mère qui situe le début de l'agression sexuelle lorsque son mari a amené pour la première fois l'enfant à la pêche; ou à l'époque où il demeurait dans un logement de quatre pièces, tandis qu'aujourd'hui, ils demeurent dans une maison.

\section{L'absence de contradiction.}

Il faut scruter la version de l'enfant pour y découvrir d'éventuelles contradictions tout en prenant garde de ne pas confondre les vraies et les fausses contradictions. Par exemple, un jeune enfant commence fréquemment par dire qu'il s'agit «d'un ami de papa» avant de nommer explicitement son père. Cette apparente contradiction s'explique par la difficulté de tout enfant à mettre en cause ses parents. Également, lorsqu'un enfant dit que cela se passait dans les toilettes puis, plus tard, que c'était au salon, s'agit-il d'une contradiction? Les événements ont très bien pu avoir lieu à plusieurs endroits et, en raison de son âge, l'enfant ne peut penser à tout en même temps. 


\section{L'augmentation progressive des agressions sexuelles.}

Il est vraisemblable de penser qu'un père agresseur sexuel augmentera au moins la fréquence de ses agressions, sinon la gravité même des actes posés. En effet, certains pères incestueux semblent avoir une obsession des seins et s'en tiennent à cette partie du corps de l'enfant, même si l'agression s'étale sur plusieurs mois, voire même plusieurs années. Mais même alors, il est probable qu'il agressera son enfant de plus en plus fréquemment.

\section{Le secret de l'agression sexuelle.}

Il y a évidemment secret lorsque cette forme de relation se continue sans être dévoilée. Du reste, l'inceste ne peut se prolonger que dans le silence. Ce secret peut être parfois imposé à l'enfant par la force ou par l'autorité du rôle : ce qu'un père fait ne peut pas être remis en question. Plusieurs facteurs contribuent à maintenir cet état des choses : la crainte de l'enfant d'être blâmé, la difficulté d'en parler avec sa mère, l'influence des avantages obtenus et des cadeaux, et la crainte que son père soit mis en prison.

\section{L'absence de modèle standard lorsqu'il y a plusieurs agresseurs.}

Bien que les actes de l'un doivent ressembler à ceux de l'autre, il existe certaines différences qu'il faut identifier.

\section{B. Les facteurs tirés de l'analyse de la forme du message :}

\section{Le message principal : l'agression sexuelle.}

Dû aux émotions intenses et au poids du secret qui lui sont rattachés, l'agression sexuelle est la plus grande préoccupation de l'enfant. Une adolescente, par exemple, consacre habituellement beaucoup plus d'ardeur à décrire l'agression qu'à nous convaincre qu'elle doit être placée pour être protégée. L'inverse serait certainement suspect. Serait également suspecte l'attitude d'un enfant qui insisterait et répéterait un même message «à dire absolument au tribunal» pour que son père soit emprisonné.

\section{L'intensité émotive.}

Entendre les premiers aveux d'un enfant victime d'inceste est très émouvant. La pression émotive nous rejoint et nous convainc. Toutefois, lorsque l'enfant a exprimé son secret, qu'il a déjà reçu compréhension et aide, il peut, par la suite, témoigner de ce qui lui arrive avec moins d'intensité apparente. Il ne faut pas alors douter pour autant de ses propos. 


\section{Lorsque l'enfant «protège» l'agresseur.}

L'enfant victime veut que l'agression sexuelle cesse, mais cela n'empêche pas qu'il puisse aimer son père. Aussi, il raconte suffisamment son histoire pour qu'on le prenne au sérieux, mais il n'essaie pas de «noircir» absolument son père. Bien au contraire, la plupart ne veulent surtout pas qu'il soit mis en prison. L'absence d'hostilité à l'égard du père, le cas échéant, donne encore plus de crédibilité aux déclarations de l'enfant.

\section{Les autres facteurs :}

En plus de l'analyse de ce que révèle l'enfant, d'autres facteurs peuvent nous guider à conclure. Par ordre croissant d'importance, on peut noter :

\section{Les problèmes de comportement.}

La masturbation compulsive, les jeux à nette connotation sexuelle, les relations sexuelles multiples et, dans une moindre mesure, les fugues, l'absentéisme scolaire, la consommation de drogue, la dépression, les tentatives de suicide, les troubles de sommeil, les troubles somatiques, etc. sont des perturbations éventuellement liées à l'inceste.

Pourtant, certains enfants vivent de tels problèmes tandis que d'autres non. Bien sûr, tout enfant ayant des problèmes de comportement n'a pas nécessairement été agressé sexuellement. Mais, les trois premiers éléments cidessus sont souvent révélateurs d'agression sexuelle.

\section{La preuve médicale.}

Il est plutôt rare qu'un examen médical puisse prouver une agression sexuelle. Évidement, une maladie transmise sexuellement ou une dilatation béante du rectum sont les traces les plus probantes d'agression sexuelle. Mais elles n'arrivent quand même pas à nommer l'agresseur!

\section{La deuxième expertise concluante.}

Il s'agit ordinairement d'une évaluation psychologique ou pédopsychiatrique de l'enfant présumé agressé sexuellement. Si l'examen est concluant et, dans la mesure où l'argumentation est solide, il faut en tenir compte.

\section{Le témoin visuel.}

Il peut arriver que l'on ait un témoin visuel. C'est ordinairement la mère qui surprend son conjoint. Cela est rare cependant. De plus, il faut être prudent 
et vérifier ce qui a été vraiment «vu», pour distinguer entre ce qu'une personne croyait avoir «vu», et qu'elle avait plutôt «interprété».

\section{L'aveu de l'agresseur.}

Évidement, lorsque l'agresseur reconnaît avoir agressé sexuellement son enfant, en donne les détails correspondant à ceux révélés par sa victime et avoue devant les membres de sa famille, il n'y a plus de doute. Malheureusement, ces aveux interviennent souvent à la toute fin du processus, alors que l'on a déjà dû décider du bien-fondé du signalement de l'agression sexuelle.

\section{LES SITUATIONS OÙ UNE GRANDE PRUDENCE S'IMPOSE}

Nous avons déjà dit combien il faut être minutieux dans l'expertise psychosociale, car toute erreur a de graves conséquences. Si on doit conclure avec prudence, il est des situations qui nécessitent plus de circonspection que d'autres, car nous risquons d'y commettre plus d'erreurs.

\section{A. Une querelle de couple.}

Si le signalement arrive alors que les parents se querellent devant les tribunaux, on peut craindre que l'enfant soit une arme utilisée contre l'autre. C'est particulièrement vrai si la garde ou les droits de visite sont en cause ${ }^{23}$.

Le plus à craindre résulte d'une mauvaise interprétation de certains gestes par ailleurs anodins. Si, par souci de l'enfant, on l'examine et le questionne «à fond» lorsqu'il revient d'une visite de chez l'autre parent, on peut réussir à «découvrir des gestes soupçonnables» où il n'y en a pas. De plus, les réactions parentales exagérées lors des «découvertes» ainsi faites peuvent biaiser l'évaluation du signalement.

23. Gijseghem, H.V. (1992). Les causes de divorce ou de droit d'accès comme contexte de la fausse allégation d'abus sexuel. Dans Gijseghem, H.V. (1992). L'enfant mis à nu. L'allégation d'abus sexuel: La recherche de la vérité. Montréal: Méridien psychologie, p.117 et suivantes. 


\section{B. Une histoire apprise.}

Lorsqu'un enfant, dès le début de la première rencontre et avant de lui avoir posé une seule question, nous révèle spontanément son «secret»: «Papa me touche les fesses», ou encore : «Papa m'a touchée ici» (en montrant son pubis), il faut recevoir l'aveu avec prudence. Le secret de l'inceste est très difficile à faire raconter, à moins que l'enfant ne réalise pas encore qu'il le subit, ou qu'il n'ait eu l'occasion d'en parler plusieurs fois déjà; ce qui du reste va compliquer l'analyse dans cette dernière hypothèse.

En effet, on peut craindre que l'enfant ait été amené à prendre parti pour un parent, surtout lorsqu'une âpre dispute existe dans le couple. À la limite, on peut même envisager qu'il s'agisse d'une histoire apprise destinée à nous la faire découvrir.

\section{Une situation invraisemblable.}

Il existe plusieurs situations inusitées et invraisemblables comme celle d'une adolescente qui soutiendrait être agressée par son père, son frère, un oncle et son grand-père tout à la fois. Ou encore, une jeune fille qui racontait que son père la caressait devant sa nouvelle conjointe et des invités, dans le salon. Dans ces cas, il faut s'appliquer à scruter tous les «détails périphériques» possibles pour découvrir la vérité.

\section{La présence de plusieurs pères.}

Dans les situations de familles reconstituées, les jeunes enfants peuvent appeler papa, le nouveau conjoint de leur mère... ou maman, la conjointe de leur père. Aussi, il faut faire préciser - surtout pour les plus jeunes - le prénom du «papa» en question ou encore demander de décrire ce «papa», en trouvant une caractéristique propre à l'identifier. Par exemple, lors d'une expertise où le signalement d'agression sexuelle s'était avéré fondé, une mère eut la mauvaise surprise de constater que le «papa» en question n'était pas son ex-conjoint - le père de la fillette - mais son conjoint actuel. Et c'est seulement par la description des vêtements du «père» et des lieux qu'on a pu identifier l'agresseur qui a finalement lui-même avoué.

\section{CONCLUSION}

Le modèle psychosocial d'une expertise dans les signalements d'inceste que nous venons d'exposer est le fruit de toute une équipe de professionnels très engagés du Centre Jeunesse de l'Estrie. Ils l'ont construit grâce à beaucoup d'efforts, de recherches, d'imagination et d'expérimentations. 
Que peut-on dire de l'efficacité de ce modèle? Malheureusement, un effort d'évaluation scientifique de son efficacité n'a pu être mené à terme. On peut quand même souligner que de tous les cas jugés fondés par le moyen de ce type d'expertise, très peu ont dû être judiciarisés. Pour les autres cas, une très nette majorité, l'agression a été reconnue verbalement et par écrit par toutes les personnes impliquées. Une entente volontaire a pu alors être conclue où toutes les parties acceptaient de collaborer avec le directeur de la protection de la jeunesse pour rétablir la situation.

En terminant, il faut souligner que ce modèle d'expertise nécessite que le directeur de la protection de la jeunesse veille à ce qu'on procure, aux intervenants qu'il mandate, une formation appropriée à ce type d'évaluation très complexe, un support clinique adéquat et une charge de cas compatible avec la disponibilité tant nécessaire ici. Souhaitons qu'on saura assurer le maintient, dans le contexte actuel, de ces conditions vitales à un travail de qualité dans le mandat si délicat et si nécessaire qu'est l'évaluation des signalements d'inceste! 